\title{
Relativistic Structure of the Nucleon Self-Energy in Asymmetric Nuclei
}

\author{
S. Ulrych and H. Müther \\ Institut für Theoretische Physik, Universität Tübingen, D-72076 Tübingen, Germany
}

\begin{abstract}
The Dirac structure of the nucleon self-energy in asymmetric nuclear matter cannot reliably be deduced from the momentum dependence of the single-particle energies. It is demonstrated that such attempts yield an isospin dependence with even a wrong sign. Relativistic studies of finite nuclei have been based on such studies of asymmetric nuclear matter. The effects of these isospin components on the results for finite nuclei are investigated.
\end{abstract}

PACS number(s): 21.60.Jz, 21.65.+f

\section{INTRODUCTION}

During the last years a substantial progress has been made in the microscopic description of the bulk properties of nuclear matter by the inclusion of relativistic features in the so-called Dirac-Brueckner-Hartree-Fock (DBHF) approximation. One finds that the self-energy of the nucleon in nuclear matter contains a large attractive component $\Sigma_{s}$ which is of the order of $-300 \mathrm{MeV}$ and transforms like a scalar under a Lorentz transformation. This very attractive contribution is compensated to a large extent by a repulsive, time-like Lorentz-vector component $\Sigma_{0}$. This partial cancellation between $\Sigma_{s}$ and $\Sigma_{0}$ leads to single-particle energies and binding energies for the nucleons, which are of the order of $-40 \mathrm{MeV}$, small compared to the nucleon rest-mass. Based on this small binding energy it has been argued for a long time that relativistic effects should be small in nuclear physics.

However, considering a self-energy with a relativistic structure as just outlined in a Dirac equation for a nucleon in a medium of nuclear matter, one finds that Dirac spinors derived from this equation exhibit a substantial enhancement of the small component as compared to the Dirac spinor of a free nucleon with the same momentum. This Dirac spinor essentially corresponds to one for a nucleon with an effective mass $m^{*}$ which is the sum of the bare mass $m$ plus the scalar part of the self-energy $\Sigma_{s}$. Assuming a value for $\Sigma_{s}$ of $-300 \mathrm{MeV}$, which is quite typical for nuclear matter at saturation density, it is obvious that this reduction of the effective mass can result in non-negligible effects. The matrix elements of the nucleon-nucleon (NN) interaction for two nucleons in nuclear matter should be evaluated employing these Dirac spinors modified by the nuclear medium, which means that the NN interaction is density-dependent. It is this density-dependence which leads to the saturation of nuclear matter in simple relativistic mean-field calculations within the Walecka model [1].

Replacing the phenomenological approach for the NN interaction used in the Walecka model by a realistic mesonexchange potential, one has to perform a nuclear structure calculation which goes beyond the mean-field or HartreeFock approximation and account for the effects of NN correlations. This can be done in a relativistic extension of the Brueckner-Hartree-Fock approximation. For that purpose one has to evaluate the $G$-matrix by solving the BetheGoldstone equation. For relativistic models of the NN interaction like the One-Boson-Exchange (OBE) potentials this Bethe-Goldstone equation corresponds to a three-dimensional reduction of the Bethe-Salpeter equation, which accounts for Pauli- and dispersive corrections due to the surrounding nuclear medium [2].

The $G$-matrix derived from this Bethe-Goldstone equation can be analysed and decomposed into five Lorentz invariant amplitudes. From these amplitudes one can calculate the nucleon self-energy within the Brueckner-HartreeFock approximation and determine its relativistic structure, which means its decomposition into a scalar term $\Sigma_{s}$, a time-like vector term $\Sigma_{0}$ and a space-like vector term $\Sigma_{v}$. This self-energy is included in a Dirac equation to determine the Dirac spinors for the nucleons. A self-consistent solution of these DBHF equations requires that the resulting spinors are used to evaluate the matrix elements of the OBE interaction and determine the $G$-matrix.

Such self-consistent DBHF calculations for nuclear matter have been performed by various groups employing different models for the NN interaction [2] 6]. All these investigations show that the relativistic effects modify the saturation properties of nuclear matter derived from realistic NN interaction within the BHF scheme. The density-dependence of the nucleon Dirac spinors yields some repulsion which increases significantly with the nuclear density. Due to this mechanism the Dirac effects of the DBHF approach supply a fine-tuning in the calculated energy of nuclear matter as a function of density, which moves the prediction for the saturation point off the well-known Coester band [7], which is obtained in non-relativistic many-body calculations of nuclear matter, towards the empirical result. Brockmann and Machleidt actually succeeded in finding a version of the Bonn potential, which fits the NN scattering data and reproduces the empirical saturation point of symmetric nuclear matter using the DBHF approach 《\. 
Various groups analyzed the relativistic structure of the $G$-matrix and derived the three components of the selfenergy, $\Sigma_{s}, \Sigma_{0}$ and $\Sigma_{v}$, in symmetric nuclear matter. They find the dependence of these three components on the momentum of the nucleon is rather weak and that the effects of the space-like vector component $\Sigma_{v}$ is rather small as compared to the other two [5,6,6,8,9. Therefore it seems to be justified that one takes advantage of this feature and derives the decomposition of the self-energy into $\Sigma_{s}$ and $\Sigma_{0}$ from the momentum-dependence of the single-particle energy $\epsilon(k)$ (see also below). In this way one can avoid the analysis of the relativistic structure of $G$, which simplifies the self-consistent procedure of DBHF significantly.

After the DBHF scheme had been applied with great success to symmetric nuclear matter, it was an obvious extension to use the same scheme for pure neutron matter and assymetric nuclear matter with various fractions of proton to neutron density as well 10 12]. In section 2, below, we would like to demonstrate that it is very dangerous to apply the simplified version of the DBHF self-consistency, i.e. deducing the Dirac components from the single-particle energies, to asymmetric nuclear systems. We will see that this scheme, which has been used e.g. by [10 13] even tends to predict the "wrong sign" for the isovector dependence of $\Sigma_{s}$ and $\Sigma_{0}$.

Attempts have also been made to apply the DBHF approach to finite nuclei as well [14 18]. One possibility in this direction is the so-called Relativistic Mean Field approach with Density dependent coupling constants (RMFD) [16,17]. The first step of this approach considers the Relativistic Mean Field or Dirac-Hartree approach for nuclear matter at a given density, adjusting an effective coupling constant for the scalar meson $\sigma$ and the vector meson $\omega$ to reproduce the results of microscopic DBHF calculations at this density. In a second step these density-dependent coupling constants are employed in a Dirac-Hartree calculation to evaluate the ground-state properties of finite nuclei in a kind of local-density approximation.

Recently this RMFD approach has been extended to asymmetric nuclear systems. Using the DBHF results of Engvik et al. [12], Shen et al. 17] determined density-dependent coupling constants for the isoscalar mesons $\sigma$ and $\omega$ and the isovector mesons $\delta$ and $\rho$ to fit the relativistic components of the self-energy for protons and neutrons at various densities and asymmetries. Since, however, this analysis is based on DBHF calculations which exhibits the "wrong sign" in the isospin structure, one obtains coupling constants for the isovector mesons in particular, which are not appropriate. In section 3 we would like to correct this sign and show the effect of a proper treatment of the isovector mesons on the structure of finite nuclei using the RMFD approach.

\section{ISOVECTOR MESONS AND ASYMMETRIC NUCLEAR MATTER}

Our investigation of the role of isovector mesons in the effective NN interaction in a nuclear medium is based on the analysis of Boersma and Malfliet [9]. They parametrize the relativistic structure of the G-matrix in terms of five Lorentz invariants

$$
G=\sum_{\alpha=1}^{5} T^{\alpha} F_{(1)}^{\alpha} F_{(2)}^{\alpha},
$$

with

$$
F_{(i)}^{\alpha}=\left\{1, \sigma^{\mu \nu}, \gamma_{5} \gamma^{\mu}, \gamma^{\mu}, \gamma_{5} \frac{\not q}{2 m^{*}}\right\}
$$

The amplitudes $T^{\alpha}$ are parametrized in terms of Yukawa functions, depending on the Mandelstam variable $t$ as

$$
T^{\alpha}=\sum_{n=1}^{4} \frac{g_{\alpha n}^{2}}{\mu_{n}^{2}-t}
$$

with effective meson masses $\mu_{n}$ and coupling constants $g_{\alpha n}$. At a given density of nuclear matter, these coupling constants are adjusted in such a way that the antisymmetrized matrix elements of the parametrization (11) - (3) reproduce the corresponding matrixelements of the $G$-matrix, calculated in the rest frame of nuclear matter [19]. More details of this parametrization can be found in [9,20]. All the results displayed in this manuscript are obtained using the parameter set III of [9,20]. Results derived from parameter sets I and II are rather similar.

Using this parametrization one can easily determine the self-energy $\Sigma^{i}(k)$ for protons and neutrons, labeled by index $i$, with momentum $k$

$$
\Sigma^{i}(k)=1 \tilde{\Sigma_{s}^{i}}(k)+\gamma \cdot \mathbf{k} \Sigma_{v}^{i}(k)-\gamma^{0} \tilde{\Sigma}_{0}^{i}(k),
$$


by solving the Dirac-Hartree-Fock equation for the NN interaction defined by the parametrization of (1) - (3) in a selfconsistent way. Inserting this self-energy into the Dirac equation for a nucleon in the nuclear medium, we obtain

$$
\left[\left(1+\Sigma_{v}^{i}(k)\right) \gamma \cdot \mathbf{k}+\left(M+\tilde{\Sigma_{s}^{i}}(k)\right)-\tilde{\Sigma_{0}^{i}}(k) \gamma^{0}\right] u_{i}(k)=\epsilon_{i}(k) \gamma^{0} u_{i}(k)
$$

Now it is convenient to eliminate $\Sigma_{v}$ and rewrite this Dirac equation into a form which only contains a scalar and a time-like vector component

$$
\left[\gamma \cdot \mathbf{k}+\left(M+\Sigma_{s}^{i}(k)\right)-\Sigma_{0}^{i}(k) \gamma^{0}\right] u_{i}(k)=\epsilon_{i}(k) \gamma^{0} u_{i}(k)
$$

where

$$
\begin{aligned}
& \Sigma_{s}^{i}=\frac{{\tilde{\Sigma_{s}^{i}}}-M \Sigma_{v}^{i}}{1+\Sigma_{v}^{i}} \\
& \Sigma_{0}^{i}=\frac{\tilde{\Sigma_{0}^{i}}-\epsilon_{i} \Sigma_{v}^{i}}{1+\Sigma_{v}^{i}}
\end{aligned}
$$

We will assume that the parametrization of $G$, which has been determined by Boersma and Malfliet for symmetric nuclear matter at various densities $\rho$ may also be used for asymmetric nuclear matter at the same density. With this assumption we ignore the fact that the $G$-matrix will not only depend on the density of nuclear matter but also on the asymmetry parameter

$$
\alpha=\frac{\rho^{p}}{\rho^{n}+\rho^{p}}
$$

where $\rho^{p}$ and $\rho^{n}$ denote the density of protons and neutrons, respectively. This implies that symmetric nuclear matter corresponds to $\alpha=0.5$ and pure neutron matter to $\alpha=0$. The assumption that the $G$-matrix does not depend on this asymmetry parameter $\alpha$ may not be sufficient for a very sophisticated study of asymmetric systems. It should be sufficient, however, for the more general remarks, which we wish to make in the present study.

As a typical example we will now first consider the case of asymmetric nuclear matter which is defined by a baryon density $\rho=0.185$ nucleon $\mathrm{fm}^{-3}$, which corresponds roughly to the saturation density of nuclear matter and an asymmetry parameter $\alpha=0.35$. Results for the self-energy of protons and neutrons are displayed in Fig. 1 as a function of the momentum $k$. One observes a momentum dependence of the scalar and vector components, $\Sigma_{s}^{r}$ and $\Sigma_{0}^{i}$ defined in (7), which is rather weak as compared to the total value of these components. The variation of these quantities as a function of momentum is around $20 \mathrm{MeV}$ for momenta below the Fermi momentum, which corresponds to about 5 percent of the total value. Therefore one may be tempted to ignore this momentum-dependence for a moment and interpret the mean value for these components

$$
U_{\beta}^{i}=\frac{\int_{0}^{k_{F i}} k^{2} \Sigma_{\beta}^{i}(k) d k}{\frac{k_{F i}^{3}}{3}},
$$

in terms of a mean field or Hartree-Fock model. The index $\beta$ in this equation represents the scalar $(\beta=s)$ or vector component $(\beta=0)$ and $k_{F i}$ stands for the Fermi momentum of protons $(i=p)$ and neutrons $(i=n)$, respectively. Assuming a meson exchange model for the NN interaction, which considers the exchange of a scalar meson $(\sigma)$ and a vector meson $(\omega)$ plus the exchange of the corresponding isovector mesons $\delta$ and $\rho$, the components of the self-energy for protons and neutrons are easily evaluated within the Hartree approximation as

$$
\begin{aligned}
U_{s}^{p} & =-\frac{g_{\sigma}^{2}}{m_{\sigma}^{2}}\left(\rho_{s}^{p}+\rho_{s}^{n}\right)-\frac{g_{\delta}^{2}}{m_{\delta}^{2}}\left(\rho_{s}^{p}-\rho_{s}^{n}\right) \\
U_{s}^{n} & =-\frac{g_{\sigma}^{2}}{m_{\sigma}^{2}}\left(\rho_{s}^{p}+\rho_{s}^{n}\right)+\frac{g_{\delta}^{2}}{m_{\delta}^{2}}\left(\rho_{s}^{p}-\rho_{s}^{n}\right) \\
U_{0}^{p} & =-\frac{g_{\omega}^{2}}{m_{\omega}^{2}}\left(\rho^{p}+\rho^{n}\right)-\frac{g_{\rho}^{2}}{m_{\rho}^{2}}\left(\rho^{p}-\rho^{n}\right) \\
U_{0}^{n} & =-\frac{g_{\omega}^{2}}{m_{\omega}^{2}}\left(\rho^{p}+\rho^{n}\right)+\frac{g_{\rho}^{2}}{m_{\rho}^{2}}\left(\rho^{p}-\rho^{n}\right)
\end{aligned}
$$


The parameter $g_{\mu}$ and $m_{\mu}$ in these equations refer to the meson-nucleon coupling constants and the masses of the varios mesons, the proton and neutron densities are represented by $\rho^{p}$ and $\rho^{n}$ and the scalar densities are defined by

$$
\rho_{s}^{i}=\frac{8 \pi}{(2 \pi)^{3}} \int_{0}^{k_{F i}} k^{2} \frac{M_{i}^{*}}{E_{i}^{*}} d k
$$

with the effective mass

$$
M_{i}^{*}=M+U_{s}^{i}
$$

and

$$
E_{i}^{*}=\sqrt{M_{i}^{*}+k^{2}}
$$

Inserting these Hartree-results for the self-energy into the Dirac eq.(6) one obtains the single-particle energy in the Hartree approximation

$$
\epsilon_{i}^{H}(k)=\sqrt{M_{i}^{*}+k^{2}}-U_{0}^{i}
$$

For nuclear matter with $\rho^{n}>\rho^{p}$ the Hartree approximation of (10) predicts more negative values for the self-energy components for neutrons, $U_{0}^{n}$ and $U_{s}^{n}$, as compared to the corresponding results for the protons. Looking again at the momentum-dependent results for these self-energy components displayed in Fig. 1 we observe that this feature is reproduced by the momentum-dependent self-energy components derived from the Groningen parametrization of DBHF. From this figure we can furthermore observe that the isospin dependence is larger for the vector component of the self-energy $\Sigma_{0}$ than for the scalar component. Translated into the Hartree analysis of eq.(10) this means that the effective $\rho$ exchange contribution to the $G$ matrix seems to be stronger than the effects of the isovector scalar meson $\delta$. This is definetely true for a realistic meson-exchange potential like the Bonn potential [2], and obviously remains valid for the $G$ matrix. The difference in the isospin dependence of $\Sigma_{s}$ and $\Sigma_{0}$ is also responsible for the single-particle energies

$$
\epsilon_{i}(k)=\sqrt{\left(M+\Sigma_{s}^{i}(k)\right)^{2}+k^{2}}-\Sigma_{0}^{i}(k)
$$

and leads to the result that the single-particle energies for the protons are more attractive than those for the neutrons (see part on the right-hand side of Fig. 1). This reflects the fact that the proton - neutron interaction is more attractive than the neutron - neutron interaction.

One of the main features of the dependence of the self-energy for protons and neutrons in asymmetric nuclear matter is displayed in the left part of Fig. 2. There we show the dependence of the average effective mass $M_{i}^{*}$ calculated according eq.(12) using the momentum averaged $U_{s}^{i}$ defined in (9) as a function of the asymmetry parameter $\alpha$. The effective mass is of course identical for protons and neutrons in the case of symmetric nuclear matter $(\alpha=0.5)$. The effective mass for the protons increases with decreasing $\alpha$ i.e. with an increasing fraction of neutrons. The effective mass for the neutrons is smaller than the corresponding $M_{p}^{*}$ for all values $0 \leq \alpha<0.5$.

This is just opposite to the behaviour observed by Engvik et al. [12,21] (see also Fig.2 of [17]) as well as Lee et al. 13]. These investigations report effective masses for the proton to be smaller than those for the neutron. One could argue that this different behaviour of $\Sigma_{s}^{i}$ or $M_{i}^{*}$ is due to the different interactions: the results of [12] have been derived from the Bonn potential A, whereas we consider parametrization of the Groningen potential. However, we would like to demonstrate that the isospin dependence of the effective mass $M_{i}^{*}$ reported in [12] is wrong and that this mistake is caused by the fact that Engvik et al. deduce the effective mass from the momentum dependence of the single-particle energy, a method which has been used by other groups before [4, 10,13.

In order to demonstrate this, we consider the momentum dependent self-energies $\Sigma_{\text {s }}^{i}(k)$ deduced from the Groningen potential and calculate the single-particle energy $\epsilon_{i}(k)$ calculated according to (14). In a next step we analyze these functions $\epsilon_{i}(k)$ and deduce from these functions effective masses $M_{i}^{*}$ and vector potentials $U_{0}^{i}$. This can be done by taking $\epsilon_{i}$ at two values of $k=k_{1}, k_{2}$ (our choice is $k_{1}=0.7 k_{F}, k_{2}=k_{F}$ ) and by adjusting the parameters $M_{i}^{*}$ and $U_{0}^{i}$ in such a way that (13) reproduces the values $\epsilon_{i}\left(k_{1}\right)$ and $\epsilon\left(k_{2}\right)$. In this way we simulate the procedure of Engvik et al. [12] and Lee et al. [13] for the Groningen potential.

The results of this procedure is displayed in the right part of Fig. 2. We find that the effective masses deduced from the momentum dependence of the single-particle energies show the same behaviour as reported by Engvik et al. [12] and Lee et al. [13]. This demonstrates very clearly that the isospin dependence dependence of the effective mass is very sensitive to the method used to determine it. Although the momentum dependence of the components of the 
self-energy $\Sigma_{0, s}^{i}$ is rather weak on the scale of the absolute values of these quantities (see discussion of Fig. 11 above), it is obviously too strong to neglect it in analyzing the momentum-dependence of $\epsilon_{i}(k)$. Therefore such an analysis leads to wrong results, in particular when we are interested in the isospin dependence.

This observation led us to compare the two schemes of analyzing the Dirac structure of the nucleon also in the case of symmetric nuclear matter. Results for the effective mass as a function of the Fermi momentum $k_{F}$ of symmetric nuclear matter are displayed in Fig. 3. The results are rather similar for densities around the empirical saturation density $\left(k_{F} \approx 1.4 \mathrm{fm}^{-1}\right)$. This suggests that the evaluation of the saturation properties of nuclear matter, as performed by Brockmann and Machleidt [4] may not be influenced too much by improving the method to determine the Dirac structure of the nucleon spinors. For other densities, however, the effective mass deduced from the momentum dependence of the single-particle energy (dashed line) deviates significantly from the effective mass derived directly from $\Sigma_{s}$. In particular at smaller densities, these are the densities which are relevant for the description of finite nuclei with medium mass, the effective mass derived from the single-particle energies is considerably smaller than the corresponding value deduced from $\Sigma_{s}$.

\section{RELATIVISTIC MEAN FIELD CALCULATIONS FOR FINITE NUCLEI}

Relativistic calculations for finite nuclei have often been based on DBHF studies of nuclear matter using a kind of local density approximation [14 18]. One possibility is to consider asymmetric nuclear matter at a given density $\rho$ and asymmetry $\alpha$ and determine the scalar and vector components $U_{s}$ and $U_{0}$ of the nucleon self-energy for protons and neutrons. Using the set of equations (10) one can determine effective coupling constants $g_{\sigma}^{2}, g_{\omega}^{2}, g_{\delta}^{2}$, and $g_{\rho}^{2}$. This means that a Dirac-Hartree calculation of nuclear matter allowing for the exchange of $\sigma, \omega, \delta$, and $\rho$ mesons with these coupling constants would reproduce the results of the DBHF calculation at this density and asymmetry. These effective coupling constants depend on density and asymmetry.

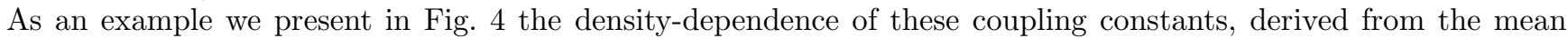
values calculated according (9). These coupling constants are represented by the solid lines and have been evaluated at an asymmetry defined by $\alpha=0.35$. The dependence of these coupling constants on $\alpha$ turns out to be weak. One can see that these effective coupling constants exhibit a moderate density-dependence. The coupling constants for the isovector mesons $\delta$ and $\rho$ are weaker than those of the isoscalar mesons.

Instead of using the momentum averaged self-energies (9) one may also try to use the corresponding quantities $U_{s, 0}^{i}$ derived from the momentum-dependence of the single-particle energies $\epsilon(k)$. Using these quantities as input to determine the effctive meson-coupling constants, we obtain the results represented by the dashed line. It must be mentioned that the coupling constans $g^{2}$ for the isovector mesons turn out to be negative in this analysis, which means that the coupling constants $g$ themselves would be imaginary. This is a consequence of the "wrong" isospin dependence of these self-energy components. The effective coupling constants derived from the single-particle spectra also show a much stronger density dependence and the absolute values for the isoscalar mesons are close to those of the isovector mesons. We would like to mention again that both sets of effective coupling constants (represented by the solid and dashed lines in Fig. (1) are determined for the same parameterization of the Groningen $G$-matrix. The only difference is that the solid line is derived from the self-energies directly, while the dashed lines are obtained from the attempt to derive these self-energies from the single-particle spectrum.

Shen et al. 17] determined effective coupling constants in the same way as we just outlined using as input data the results of Engvik et al. 12. This means that they start from components $U_{s, 0}^{i}$ which are derived from the singleparticle spectrum $\epsilon(k)$ calculated for the Bonn potential. It is remarkable that the coupling constants derived in 17] show features very similar to those represented by the dashed line above. The coupling constants $g^{2}$ are negative for the isovector mesons (note that the isovector components in eq.(6) of 17] have a wrong sign) and all effective coupling constants show a strong density dependence, very similar to the dashed lines in our Fig. 4 .

Considering a fixed asymmetry parameter $\alpha$ one may now consider the various coupling constants, which for the asymmetry assumed just depend on the total baryon density and solve the Dirac-Hartree or relativistic mean field equations assuming a local density approximation for the meson-nucleon coupling constant. Details of the procedure have been presented in [15]. Note that we do not include the effect of rearrangement terms [17].

Results for such Dirac Hartree calculations with density dependent meson nucleon coupling constants are listed in Tab. I. As examples we consider the ground-state properties of the nuclei ${ }^{16} \mathrm{O}$ and ${ }^{22} \mathrm{O}$. Three different models are considered. (a): a pure $\sigma-\omega$ model, the density dependent coupling constants for these two mesons are derived from the results of symmetric nuclear matter. (b): a $\sigma, \omega, \rho-\delta$ model in the limit of symmetric matter, i.e. $\alpha=$ 0.5. The density dependent coupling constants for these mesons are obtained by analyzing the proton and neutron self-energies for asymmetric nuclear matter, extrapolated to the symmetric case of $\alpha=0.5$. (c): a $\sigma, \omega, \rho-\delta$ model for asymmetric nuclear matter at $\alpha=0.36$, which corresponds the proton fraction of ${ }^{22} O$. For each of these models 
(a) - (c) the coupling constants derived either from the relativistic structure of the self-energy (results listed in the left part of the table) or from the momentum dependence of the single-particle energies (results in the right part).

One can see from these results that the two methods to determine the density dependence of the effective meson coupling constants lead to quite different results even for the isospin symmetric system ${ }^{16} O$. The difference in the calculated binding energy is as large as $0.6 \mathrm{MeV}$ per nucleon. The deviation of the second scheme from the first one is even larger for the non-symmetric nucleus ${ }^{22} \mathrm{O}$. Note that the differences between the two methods, around $0.9 \mathrm{MeV}$ per nucleon, is larger than the effect of the isovector mesons: the difference between the $\sigma-\omega$ model and the models including isovector mesons is only around $0.3 \mathrm{MeV}$ per nucleon.

\section{CONCLUSIONS}

The accuracy of the simple technique which tries to extract the Dirac structure of the nucleon self-energy in nuclear matter from the momentum dependence of the single-particle energy [4, 10, 13] has been investigated using the relativistic parametrization of the $G$-matrix of Boersma et al. [9]. It is demonstrated that the analysis based on the single-particle spectrum fails if asymmetric nuclear systems are considered. The isovector components of the self-energy derived in this way exhibit even the wrong sign. The simplified analysis reproduces the structure of the self-energy around the saturation density but overestimates the scalar part of the self-energy at smaller densities. Therefore the improvement of this scheme may not have a very significant effect on the calculated saturation property of symmetric nuclear matter 4 . A more apropriate analysis of the Dirac structure of the nuclear self-energy is required to derive density-dependent effective coupling constants to be used in Dirac-Hartree calculations of finite nuclei.

This work has been supported by the "Graduiertenkolleg Struktur und Wechselwirkung von Hadronen und Kernen" (DFG GRK 132/2).

[1] B.D. Serot and J.D. Walecka, Adv. Nucl. Phys. 16, 1 (1986)

[2] R. Machleidt, Adv. Nucl. Phys. 19, 189 (1989)

[3] M.R. Anastasio, L.S. Celenza, W.S. Pong, and C.M. Shakin, Phys. Rep. 100, 327 (1983)

[4] R. Brockmann and R. Machleidt, Phys. Lett. B149, 283 (1984)

[5] B. Ter Haar and R. Malfliet, Phys. Rep. 149, 207 (1987)

[6] H. Huber, F. Weber, and M.K. Weigel, Phys. Lett. B317, 485 (1993)

[7] F. Coester, S. Cohen, B.D. Day and C.M. Vincent, Phys. Rev. C1, 769 (1970)

[8] H. Elsenhans, H. Müther, and R. Machleidt, Nucl. Phys. A515, 715 (1990)

[9] H.F. Boersma and R. Malfliet, Phys. Rev. C49, 233 (1994), Erratum C50, 1253 (1994)

[10] H. Müther, M. Prakash, and T.L. Ainsworth, Phys. Lett. B199, 469 (1987)

[11] L. Engvik, M. Hjorth-Jensen, E. Osnes, G. Bao and E. Østgaard, Phys. Rev. Lett. 73, 2650 (1994)

[12] L. Engvik, E. Osnes, M. Hjorth-Jensen, G. Bao and E. Østgaard, Ap. J. 469, 794 (1996)

[13] C.-H. Lee, T.T.S. Kuo, G.Q. Li, and G.E. Brown, preprint nucl-th/9703034

[14] H. Müther, R. Machleidt, and R. Brockmann, Phys. Rev. C42, 1981 (1990)

[15] R. Fritz and H. Müther, Phys. Rev. C49, 633 (1994)

[16] R. Brockmann and H. Toki, Phys. Rev. Lett. 68, 3408 (1992)

[17] H. Shen, Y. Sugahara, and H. Toki, Phys. Rev. C55, 1211 (1997)

[18] H.F. Boersma and R. Malfliet, Phys. Rev. C49, 1495 (1994)

[19] F. de Jong, Thesis, Groningen 1992, Phys. Rev. C44, 998 (1991)

[20] H.F. Boersma, Thesis, Groningen 1992

[21] L. Engvik, M. Hjorth-Jensen, private communication 


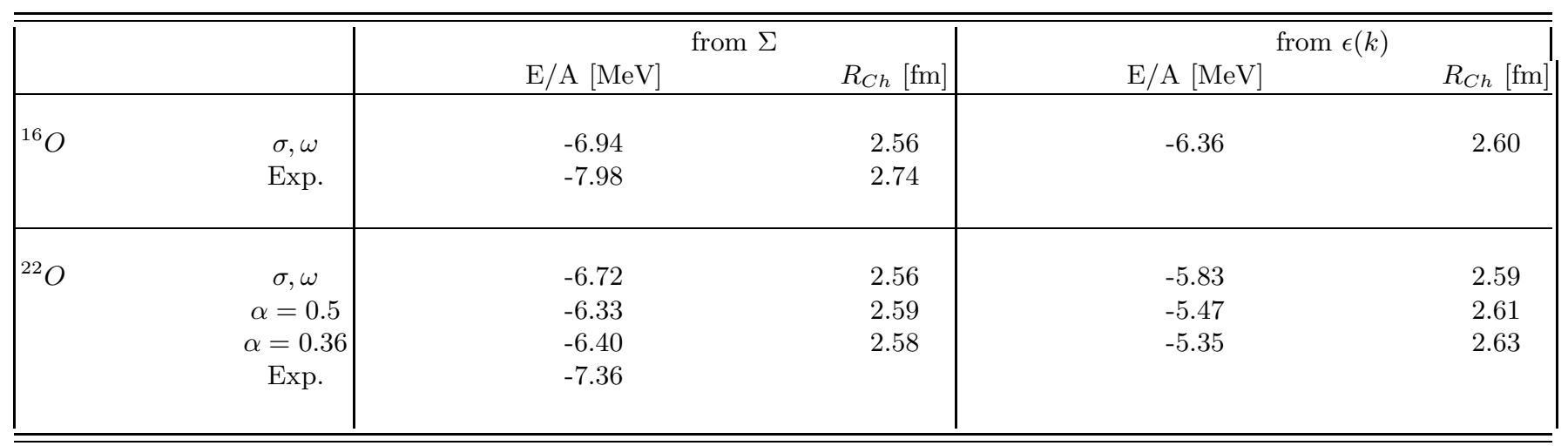

TABLE I. Energy per nucleon (E/A) and radius of the charge distribution $R_{C h}$ calculated in various versions of the Dirac Hartree approach with density dependent coupling constants. The calculated energy has been corrected to account for the effects of the spurious center of mass motion. Details of the various approaches are discussed in the text. 


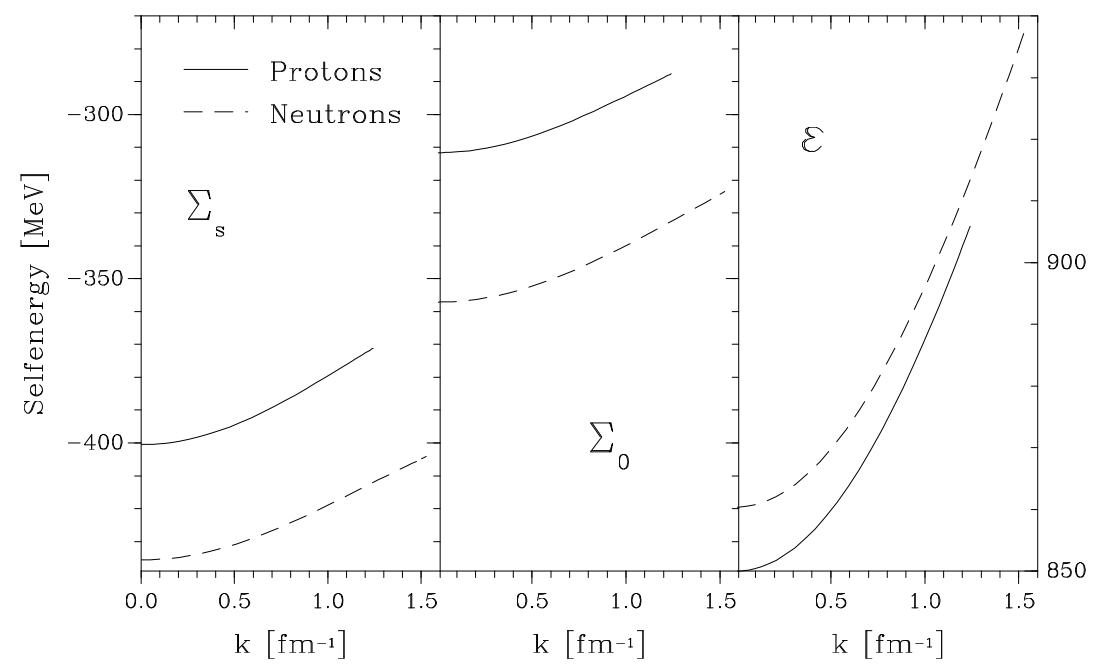

FIG. 1. Scalar $\left(\Sigma_{s}\right.$, left part) and vector component $\left(\Sigma_{0}\right.$, middle part) of the self-energy and the single-particle energy $(\epsilon$, see (14 ), right part of the figure) for protons (solid line) and neutrons (dashed line) as a function of momentum $k$. Results are displayed for asymmetric nuclear matter with a baryon density $\rho=0.185$ nucleon $\mathrm{fm}^{-3}$ and an asymmetry parameter $\alpha=$ 0.35 .

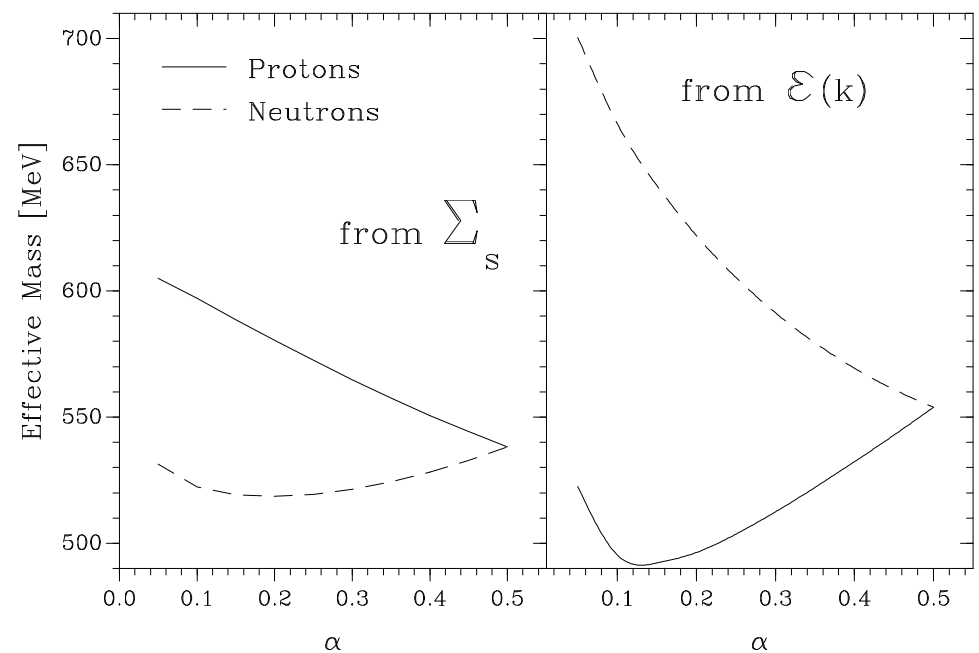

FIG. 2. Effective masses of nuclear matter $\left(\rho=0.185\right.$ nucleon $\left.\mathrm{fm}^{-3}\right)$ as a function of the asymmetry parametr $\alpha$. The effective masses displayed in the left part of the figure have been derived from the momentum averaged self-energy $\Sigma_{s}$ of eq.(9), while those in the right part of the figure are deduced from the single-particle energies $\epsilon(k)$ according eq. 13 ). 


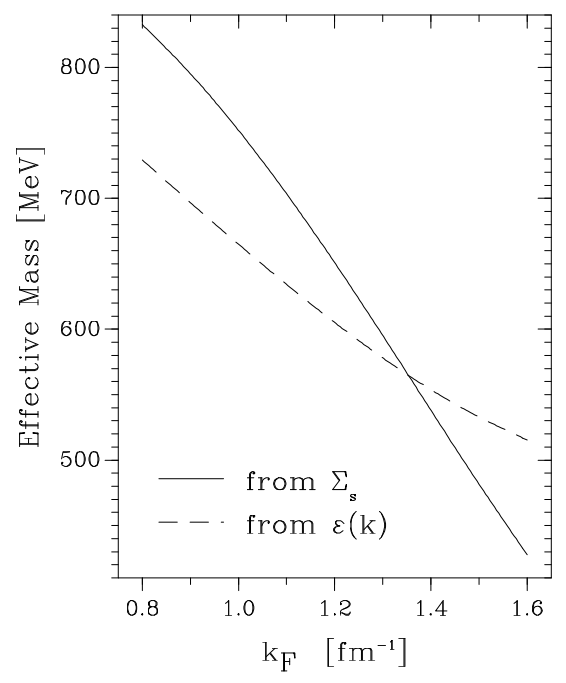

FIG. 3. Effective masses of symmetric nuclear matter as a function of the Fermi momentum $k_{F}$. The results represented by the solid line have been derived from the momentum averaged self-energy $\Sigma_{s}$ of eq.(9). Those shown by the dashed line are deduced from the single-particle energies $\epsilon(k)$ according eq.(13).

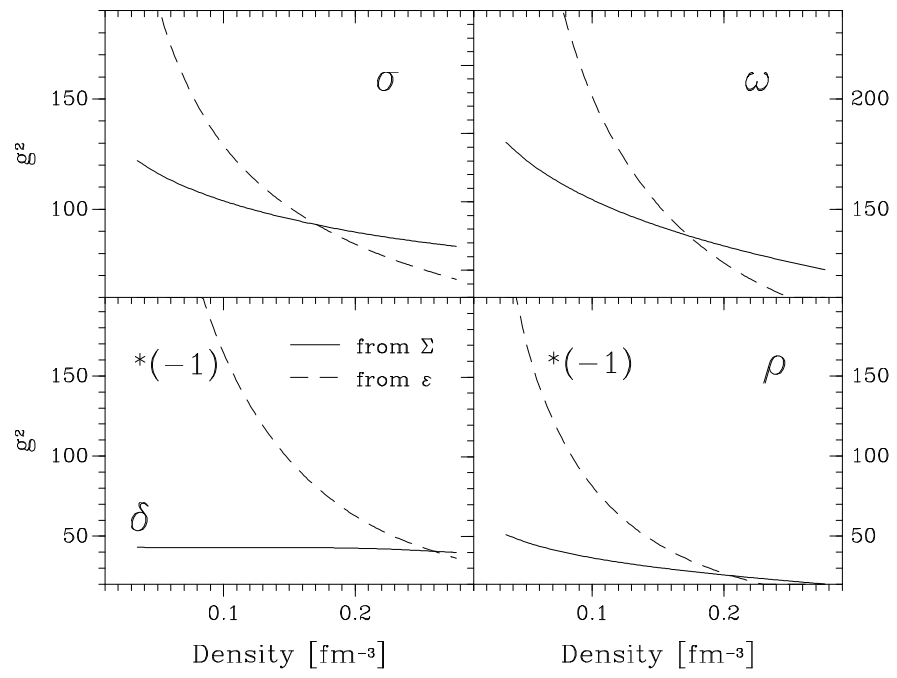

FIG. 4. Effective coupling constants to be used in Dirac-Hartree calculations as a function of density. These coupling constants are derived from (10) assuming meson masses of $550 \mathrm{MeV}, 783 \mathrm{MeV}, 983 \mathrm{MeV}$, and $769 \mathrm{MeV}$ for $\sigma$, $\omega$, $\delta$, and $\rho$ mesons, respectively. The solid lines are derived from the momentum averaged self-energies, while the dashed lines are obtained using effective masses derived from the single-particle spectrum $\epsilon(k)$. Note that the coupling constants $g_{i}^{2}$ are negative for the isovector mesons if they are derived from $\epsilon(k)$. 